\title{
QCD in magnetic fields: \\ from Hofstadter's butterfly to the phase diagram
}

\section{Gergely Endrődi ${ }^{1}$}

I revisit the problem of a charged particle on a two-dimensional lattice immersed in a constant (electro)magnetic field, and discuss the energy spectrum - Hofstadter's butterfly - from a new, quantum field theoretical perspective. In particular, I point out that there is an intricate interplay between a) the structure of the butterfly at low magnetic flux, b) the absence of asymptotic freedom in QED and c) the enhancement of the quark condensate by a magnetic field at zero temperature. I proceed to discuss the response of the QCD condensate to the magnetic field at nonzero temperatures in four space-time dimensions, present the resulting phase diagram and compare it to low-energy model predictions.

The XXXII International Symposium on Lattice Field Theory

June 23 - 28, 2014

New York, NY, USA.

\footnotetext{
${ }^{1}$ Institute for Theoretical Physics, University of Regensburg, D-93040 Regensburg, Germany.
} 


\section{Acknowledgments}

It is a great honor to be selected to receive this year's Ken Wilson prize for significant contributions to our understanding of QCD matter in strong magnetic fields and to QCD thermodynamics. This recognition urges me to keep up doing good research and to contribute to our field as significantly as I possibly can. I have not been so lucky to meet Kenneth G. Wilson in person, but reading through a collection of anecdotes [1], I feel that I would have greatly enjoyed interacting with him. One of his quotes I find particularly appropriate and useful as a guideline:

You shouldn't choose a problem on the basis of the tool. You start by thinking about the physics problem ... maybe you'll solve it using computer techniques, maybe using a contour integral; but it's very important to approach it starting from the physics because otherwise you get lost in the use of the tool, and lose track of where you're trying to go.

Although most problems in lattice gauge theory are more likely to be solved by a supercomputer than by contour integration, I still believe it is important to bear this principle in mind. This is one of the very reasons I find QCD in magnetic fields so fascinating: besides the problems requiring largescale numerical simulations, various aspects of the topic also allow for an analytical treatment. We will see examples for both during the talk.

At this point I would like to express my gratitude to my collaborators; this research would not have been possible without their help. I would especially like to thank my colleagues and friends with whom I collaborated on magnetic field-related topics: Gunnar Bali, Falk Bruckmann, Martha Constantinou, Marios Costa, Zoltán Fodor, Sándor Katz, Tamás Kovács, Stefan Krieg, Haris Panagopoulos, Andreas Schäfer and Kálmán Szabó. I am also grateful for many enlightening discussions with Jens Oluf Andersen, Szabolcs Borsányi, Pavel Buividovich, Massimo D’Elia, Gerald Dunne, Eduardo Fraga, Christof Gattringer, Antal Jakovác, Claudia Ratti, Marco Ruggieri, Hans-Peter Schadler, Andreas Schmitt, Igor Shovkovy and Bálint Tóth.

\section{Introduction: butterflies and lattices}

The physics of Quantum Chromodynamics (QCD) in the presence of background magnetic fields is remarkably rich. The most interesting aspects are how the magnetic field breaks rotational symmetry inducing anisotropic pressures; how it affects chiral symmetry breaking and deconfinement; and how it modifies various hadronic properties. Besides these challenging theoretical concepts, background magnetic fields in QCD have various applications including the cosmology of the early universe, non-central heavy-ion collisions and magnetized neutron stars [2].

A particularly beautiful example for the complexity that magnetic fields can induce is provided by Hofstadter's butterfly [3]: the energy spectrum of a Bloch electron immersed in a background magnetic field, see Fig. 1. The plot summarizes the quantum mechanically allowed energy levels (on the horizontal axis) for various values of the magnetic flux (on the vertical axis). The butterfly exhibits an apparently recursive hierarchy, with the coarse structure repeated on finer levels in ever smaller copies. A hint towards understanding this recursive pattern is provided by considering two aspects of the problem separately: the interaction of the electron with the infinite periodic lattice potential (with lattice spacing $a$ ), and its coupling to the background magnetic field $B$. The former 
problem gives rise to periodic Bloch waves, whereas the latter is described in terms of Landau levels. Both aspects involve a typical frequency [3]: In the non-relativistic treatment of a particle with mass $m$ and charge $q$, Landau levels are characterized by the cyclotron frequency $q B / m$, while the frequency of the Bloch wave with maximal momentum is $(2 \pi / a) /(m a)$. The ratio of these two characteristic frequencies equals

$$
\alpha \equiv a^{2} q B /(2 \pi),
$$

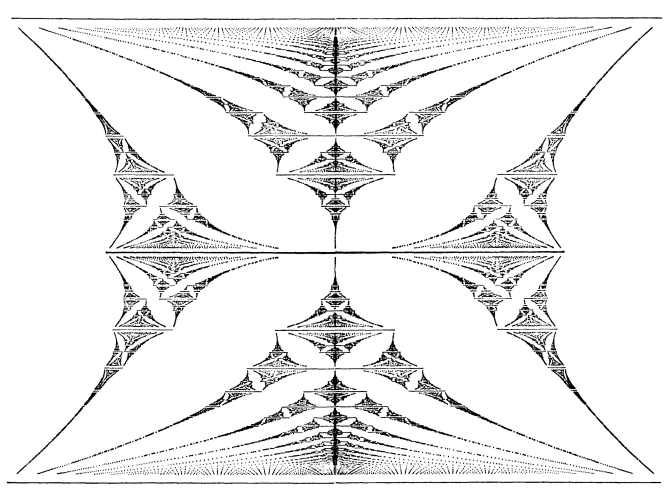

Figure 1: Hofstadter's butterfly [3].

which is proportional to the magnetic flux through an elementary plaquette of the lattice. It turns out that if the characteristic frequencies are commensurable (i.e., if $\alpha \in \mathbb{Q}$ ), the energy eigenvalues accumulate into finite bands, separated by finite gaps. In contrast, if the involved frequencies are incommensurable $(\alpha \notin \mathbb{Q})$ the energy spectrum dissolves into a zero-measure nowhere dense set that is isomorphic to the Cantor set $[3,4]$. In some sense the two problems are in this case incompatible with each other, and the particle becomes unable to obey both the periodic structure of the Bloch wave and the circular structure of the Landau levels. This frustration manifests itself in the fractal structure of the butterfly.

In the present talk, this solid state physics problem will be interpreted from a new, quantum field theoretical (QFT) point of view. Specifically, I will consider the QCD vacuum exposed to background magnetic fields in the lattice regularization. As we will see, the eigenvalue spectrum of this problem, for vanishing gauge coupling in two dimensions, coincides with the energy levels of the solid state physics example. On the one hand, the original 'solid state physics butterfly' is obtained in terms of a physical lattice spacing, characteristic to the crystal on which the electron lives. On the other hand, for the 'QFT butterfly' the lattice spacing plays the role of a regulator. This regulator (after renormalization is performed) is removed from the theory via the continuum limit $a \rightarrow 0$. In this limit the lattice structure disappears, which, in turn, implies that the 'QFT butterfly' is merely a lattice artefact. Still, as will be argued below, certain aspects of the butterfly do survive the $a \rightarrow 0$ limit and correspond to concepts in continuum physics. After pointing out these aspects, I will generalize the discussion to full dynamical QCD in four space-time dimensions, and discuss the phase diagram in the magnetic field - temperature plane using non-perturbative lattice simulations.

\section{Magnetic field, spectrum and symmetries}

\subsection{In the continuum}

The interaction with the background magnetic field proceeds via minimal coupling, with a Landau-gauge electromagnetic potential $A_{\mu}$,

$$
D_{\mu}=\partial_{\mu}+i q A_{\mu}, \quad A_{y}=B x, \quad A_{v}=0, \quad v \neq y,
$$

where $q$ denotes the charge of the particle and the coordinate system was oriented such that the magnetic field points in the positive $z$ direction. For charged bosons, the equation of motion involves the Klein-Gordon operator $D^{2}$, whereas for charged fermions, the Dirac operator $\not D=\gamma_{\mu} D_{\mu}$, 
where $\gamma_{\mu}$ denote the Euclidean $\gamma$-matrices. Besides the coupling to the magnetic field, there are no other interactions: this is what I will refer to below as the 'free' case.

The eigenvalues of $\sqrt{-D^{2}}$ and $i \not D$, respectively, give the allowed energy levels in the bosonic and fermionic problems. Simple $\gamma$-matrix identities show that the two operators are related to each other via

$$
\not D^{2}=D^{2}+\sigma \cdot q B, \quad \sigma \equiv \frac{1}{2 i}\left[\gamma_{x}, \gamma_{y}\right] .
$$

The three operators $\not D^{2}, D^{2}$ and $\sigma$ pairwise commute with each other, and thus have common eigenvectors. Eq. (3.2) acting on each joint eigenvector produces a relation between the eigenvalues, written compactly as

$$
\operatorname{Spec}\left\{\not D^{2}\right\}=\operatorname{Spec}\left\{D^{2}\right\}+\operatorname{Spec}\{\sigma\} \cdot q B .
$$

The eigenvalues of all three operators can be found in a straightforward way. The solutions are written in terms of Landau levels indexed by a non-negative integer $n$ and the component $s= \pm 1 / 2$ of the spin in the direction of the magnetic field. They indeed fulfill Eq. (3.3):

$$
\operatorname{Spec}\left\{\not D^{2}\right\}=-2 q B(n+1 / 2+s), \quad \operatorname{Spec}\left\{D^{2}\right\}=-2 q B(n+1 / 2), \quad \operatorname{Spec}\{\sigma\}=2 s .
$$

\subsection{On the lattice}

On the lattice, the gauge potential (3.1) is implemented through $\mathrm{U}(1)$ phases $u_{\mu}(n)$ that live on the links between lattice sites,

$$
u_{y}(n)=\exp \left(i a^{2} q B n_{x}\right), \quad u_{x}(n)=\exp \left(-i a^{2} q B N_{x} n_{y} \cdot \delta_{n_{x}, N_{x}-1}\right), \quad u_{z}(n)=u_{t}(n)=1,
$$

where the sites are labeled by integers $n=\left(n_{x}, n_{y}, n_{z}, n_{t}\right)$, with $n_{\mu}=0 \ldots N_{\mu}-1$ and $a$ is the lattice spacing. For convenience, the lattice extents $N_{\mu}$ are taken to be even integers. The 'twist' of the $x$-links at $n_{x}=N_{x}-1$ is necessary to satisfy periodic boundary conditions for the gauge potential and to ensure the constancy of the magnetic field throughout the $x-y$ plane [5]. In this setup, the flux of the magnetic field is quantized [6],

$$
q B \cdot a^{2} N_{x} N_{y}=2 \pi N_{b}, \quad N_{b} \in \mathbb{Z}, \quad 0 \leq N_{b}<N_{x} N_{y} .
$$

Thus, there is a minimal magnetic field due to the finiteness of the volume. In addition, due to the periodicity of the links (3.5) in $N_{b}$, there is also a maximal possible magnetic field, which is set by the square of the inverse lattice spacing. In terms of $N_{b}$, Hofstadter's parameter (2.1) reads

$$
\alpha=N_{b} /\left(N_{x} N_{y}\right), \quad 0 \leq \alpha \leq 1 .
$$

This implies that on a finite lattice $\alpha$ is always rational, and the true fractal butterfly only emerges in the infinite volume limit $N_{x} N_{y} \rightarrow \infty$. In this limit, an irrational value of $\alpha$ is obtained as the ratio of two integers, both of which approach infinity. Similarly, the continuous bands at $\alpha \in \mathbb{Q}$ are only present in the infinite volume limit and are approached by the discrete spectra on finite lattices.

Next, we have to specify the lattice discretization of the operator appearing in the equation of motion. For the bosonic case, the simplest discretization is

$$
D_{n m}^{2}=\frac{1}{a^{2}} \sum_{\mu}\left[u_{\mu}(n) \delta_{m, n+a \hat{\mu}}+u_{\mu}^{\dagger}(n-a \hat{\mu}) \delta_{m, n-a \hat{\mu}}-2 \delta_{m, n}\right] .
$$


In the fermionic case, the most convenient discretization is the staggered formulation. Here, the $\gamma$-matrices are replaced by space-dependent phases $\eta_{\mu}$, which result from a local transformation of the fermion field that diagonalizes the action in spinor space. The resulting lattice Dirac operator, again in the presence of the $\mathrm{U}(1)$ phases Eq. (3.5) is written as

$$
\not D_{n m}=\frac{1}{2 a} \sum_{\mu}\left[u_{\mu}(n) \eta_{\mu}(n) \delta_{m, n+a \hat{\mu}}-u_{\mu}^{\dagger}(n-a \hat{\mu}) \eta_{\mu}(n-a \hat{\mu}) \delta_{m, n-a \hat{\mu}}\right], \quad \eta_{\mu}(n)=(-1)^{\sum_{v=x}^{\mu-1} n_{v}} .
$$

In the following, the discussion will be restricted to two spatial dimensions, such that the sums in Eqs. (3.8) and (3.9) include $\mu=x$ and $y$.
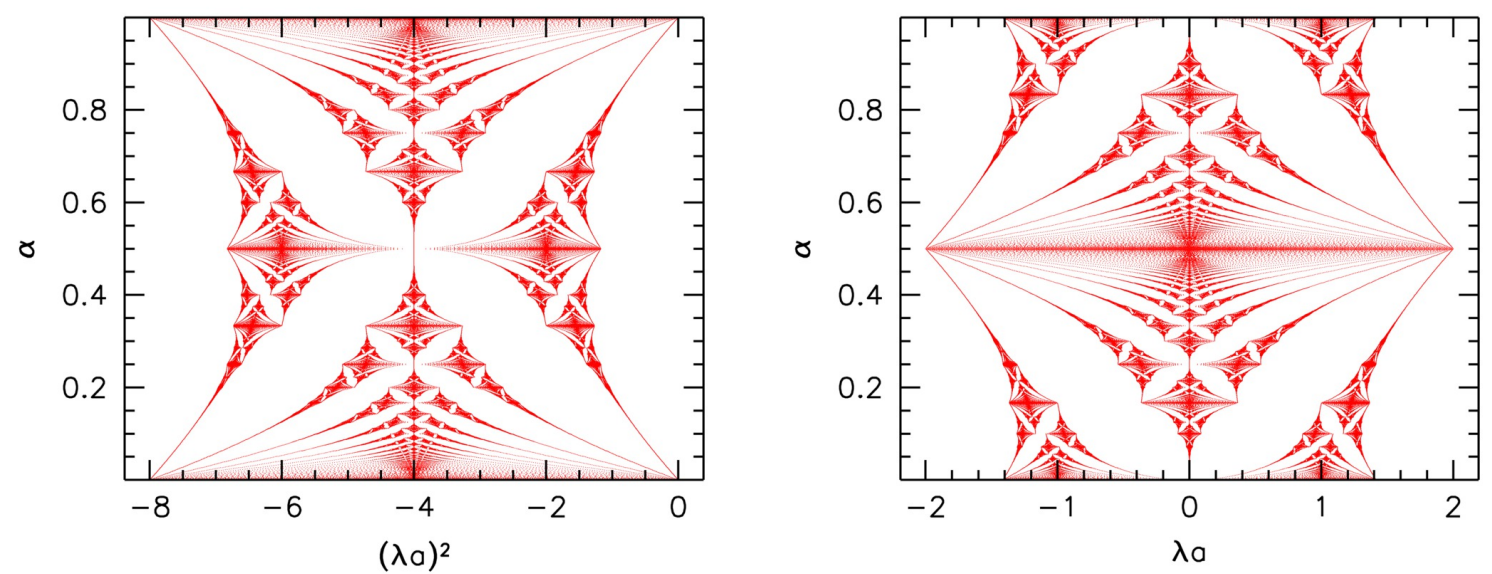

Figure 2: Butterfly in the bosonic (left panel) and fermionic (right panel) cases. The eigenvalues in lattice units are plotted against the strength of the magnetic field.

The lattice eigenvalues of $i \not D$ and those of $D^{2}$ can be found numerically by diagonalizing the corresponding matrices (note that both of these operators are Hermitean and thus have a real spectrum). A lattice of size $N_{x}=N_{y}=40$ is considered and the Lapack library is used to determine all eigenvalues. The results are shown in Fig. 2 for the bosonic and fermionic cases. Apparently, the two spectra are related to each other by a simple transformation (a translation by four and a rescaling by two) as well as by a shift in the magnetic field parameter $\alpha$,

$$
\operatorname{Spec}\left\{D^{2}(\alpha)\right\}=2 \cdot \operatorname{Spec}\{i \not D((\alpha+1 / 2) \bmod 1)\}-4 .
$$

This relation can be proven analytically by exploiting the exact form of the operators (3.8) and (3.9) after inserting the links (3.5) and using the fact that $N_{x}$ is even. Specifically, the staggered phases $\eta_{x}=1, \eta_{y}=\exp \left(i \pi n_{x}\right)$ induce the shift in the magnetic field by one half of the period, cf. Eq. (3.5). The sign in front of the adjoint links (positive in the bosonic and negative in the fermionic case) can be gauged away and does not modify the spectrum ${ }^{1}$. Further symmetries of the spectra are

$$
\operatorname{Spec}\{i \not D(\alpha)\}=\operatorname{Spec}\{i \not D(1-\alpha)\}, \quad \operatorname{Spec}\{i \not D(\alpha)\}=\operatorname{Spec}\{-i \not D(\alpha)\},
$$

${ }^{1}$ The corresponding local $\mathrm{U}(1)$ gauge transformation reads

$$
\psi(n) \rightarrow i^{n_{x}+n_{y}} \psi(n),
$$

which effectively multiplies all links in the $x-y$ plane by $i$ and, thus, flips the sign of the adjoint links and produces an overall factor $i$. Note that this is only true if $N_{x}$ and $N_{y}$ are both multiples of four. This constraint, however, is a boundary effect, which disappears in the infinite volume limit. A similar argument was discussed in Ref. [7]. 
which follow from the periodicity of the $\mathrm{U}(1)$ links in $\alpha$, parity symmetry and chiral symmetry. The operator discussed by Hofstadter [3] was $D^{2}$, i.e. the original butterfly ${ }^{2}$ actually describes scalar particles and corresponds to the spectrum in the left panel of Fig. 2 (cf. Fig. 1). Nevertheless, through the symmetry (3.10), the fermionic and bosonic spectra are in a one-to-one correspondence to each other. It is important to mention that the butterfly is (to a certain extent) also accessible in experiments: the electrical conductivity of graphene samples was found to exhibit a fractal pattern in magnetic fields [8]

\section{Correspondence between continuum and lattice}

Let me proceed by pointing out the differences and similarities between the continuum and lattice spectra. As was mentioned in the introduction, in the quantum field theoretical setting the lattice spacing is a mere regulator that is eliminated in the continuum limit. Correspondingly, the butterfly must disappear as $a \rightarrow 0$. Indeed, at fixed magnetic field $B$ the parameter $\alpha$ approaches zero in this limit and, thus, only the low- $\alpha$ end of the spectrum may play a physical role. Therefore, it is instructive to compare the lattice and continuum settings in this region.

In the continuum, the energy eigenvalues obey the Landau-level structure Eq. (3.4). In particular, the lowest levels are given by

$$
\text { fermions: } \quad(\lambda a)^{2}=q B \cdot(0,2, \ldots), \quad \text { bosons: } \quad(\lambda a)^{2}=q B \cdot(1,3, \ldots) \text {. }
$$

In the low- $\alpha$ region, the lattice eigenvalues indeed follow these continuum energies, see Fig. 3 , where the lower half of the fermionic butterfly is shown. The fermionic Landau levels are the curves starting from the origin in the plot. In addition, combining the symmetries (3.10) and (3.12) also reveals that the vertical reflection of the fermionic butterfly coincides (up to a simple linear rescaling) with the bosonic butterfly. Therefore, the bosonic Landau levels also show up in the same diagram, starting at the upper corners of Fig. 3. Notice that while the fermionic levels (the eigenvalues of $i \not D$ ) are proportional to $\sqrt{q B}$, the bosonic levels (the eigenvalues of $D^{2}$ ) are proportional to $q B$. As a result, the fermionic curves are quadratic, while the bosonic ones linear in the figure. Note moreover that the lowest fermionic Landau level is independent of $B$.

As the magnetic flux increases, the lattice eigenvalues tend to deviate from the continuum curves, dissolve into bands and mix with each other, forming the recursive

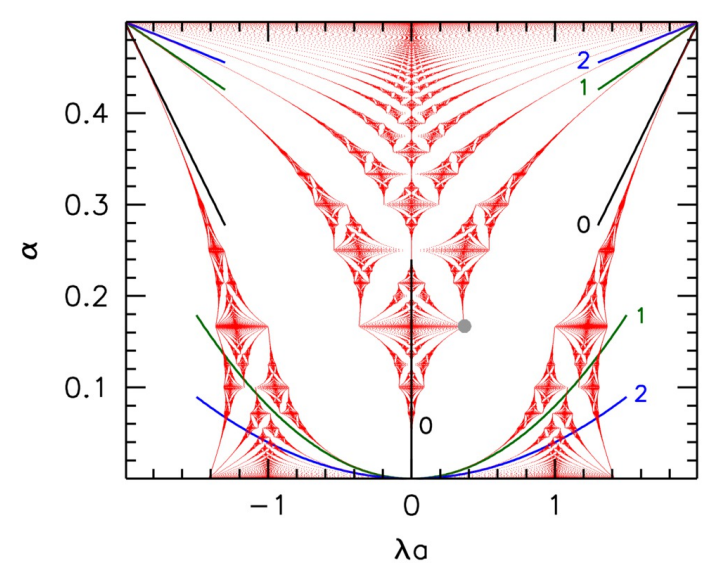

Figure 3: The butterfly as a labyrinth of fermionic (solid lines starting at the origin) and bosonic (solid lines starting at the upper corners) continuum Landau levels. The finer structure is generated by recursively appearing smaller copies of this skeleton - see a zeroth fermionic Landau level starting at the gray dot.

\footnotetext{
${ }^{2}$ Even though the feedback of several people in the audience showed that the fermionic spectrum does not resemble an actual butterfly, in the absence of a spot-on alternative I will stick to this nomenclature.
} 
pattern. Notice that this breaking up of the continuum curves proceeds in an apparently similar fashion for all Landau levels. Therefore, the 'coarsest' structure of the butterfly is provided by continuum Landau levels, which are all similar to each other and also get repeated on the finer levels. Notice, for example, a sub-structure zeroth Landau level starting in the middle of the figure, indicated by the gray dot. This is a new (qualitative) representation of Hofstadter's butterfly as a labyrinth of hierarchically embedded fermionic and bosonic Landau levels.

\subsection{The quark condensate}

Besides the difference of the role played by the lattice spacing, there is another important difference between the 'solid state butterfly' and the 'QFT butterfly'. Namely, the energy of a single electron is in principle measureable, while one eigenvalue of $i \not D$ has no physical meaning. Instead, physical observables are typically obtained by combining all eigenvalues of the Dirac operator into a spectral sum. One of the most important observables in QCD is the quark condensate, which can be written in such a spectral representation as

$$
\bar{\psi} \psi^{2 \mathrm{D}} \equiv \frac{1}{V_{2}} \operatorname{tr}(\not D+m)^{-1}=\frac{1}{V_{2}} \sum_{j} \frac{m}{\lambda_{j}^{2}+m^{2}},
$$

where $m$ denotes the mass of the quark and $V_{2}=a^{2} N_{x} N_{y}$ the two-dimensional volume. In addition, the change of the condensate induced by the magnetic field is defined as

$$
\left.\Delta \bar{\psi} \psi^{2 \mathrm{D}} \equiv \bar{\psi} \psi^{2 \mathrm{D}}\right|_{B}-\left.\bar{\psi} \psi^{2 \mathrm{D}}\right|_{B=0} .
$$

For each value of the magnetic field (i.e. for each $\alpha$ ) the condensate is calculated from the corresponding lattice eigenvalues according to Eq. (4.2). The so obtained condensate difference is plotted in Fig. 4 for two intermediate values of the mass in the low- $\alpha$ region. At masses much larger than the typical differences between the eigenvalues, the dependence of $\Delta \bar{\psi} \psi^{2 \mathrm{D}}$ on $\alpha$ is completely smooth, since the mass washes out the irregular changes in the eigenvalues as $\alpha$ is tuned. As $m$ is reduced, more and more of the fractal pattern of the butterfly becomes visible, revealed by oscillatory segments that appear in the condensate. In the chiral limit the oscillations
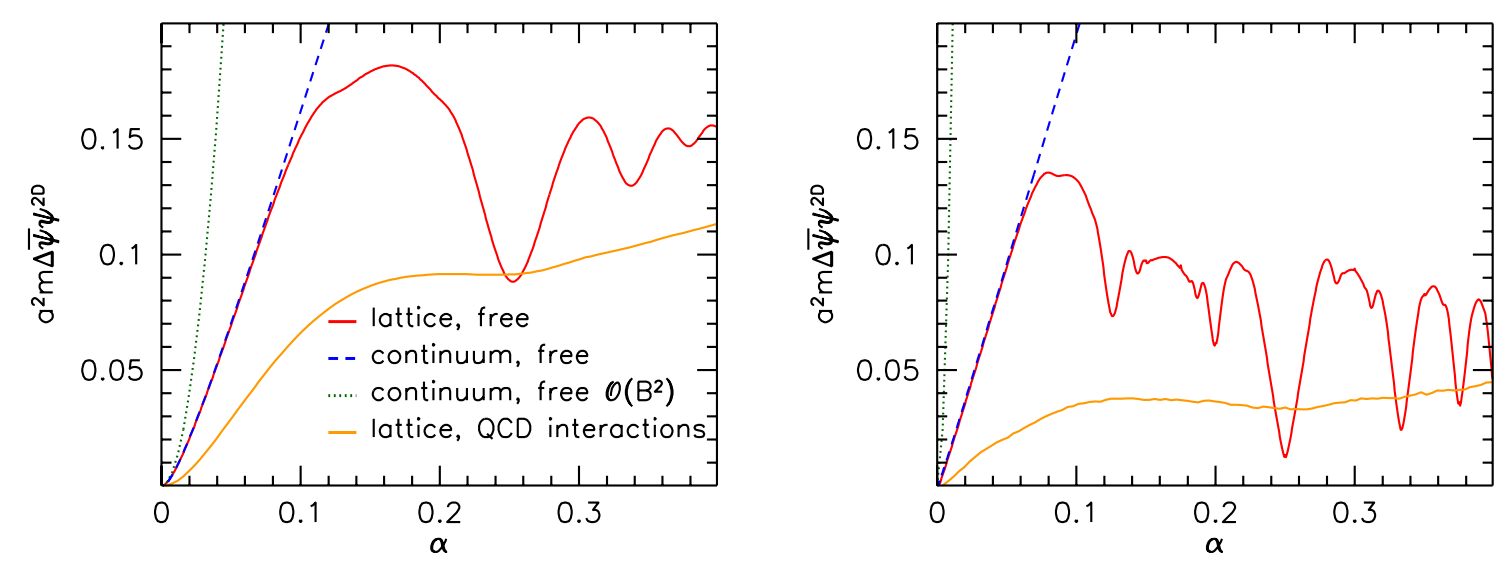

Figure 4: The quark condensate on the lattice (solid red line) at two intermediate values of the mass ( $m a=$ 0.2048 in the left panel and $m a=0.0512$ in the right panel). Also indicated are the continuum condensate (blue dashed line), the quadratic contribution to $\Delta \bar{\psi} \psi^{2 \mathrm{D}}$ (green dotted line) and the condensate calculated on the lattice in the presence of QCD interactions (solid orange line). 
approach a fractal curve that fully explores the recursive structure of the butterfly. A simple demonstration that was presented during the talk to illustrate the dependence of $\Delta \bar{\psi} \psi^{2 \mathrm{D}}$ on the mass and on the magnetic field can be found at the url [9].

The condensate can also be calculated using the continuum energies Eq. (3.4). The result see Eq. (A.8) in App. A - is indicated in Fig. 4 by the blue dashed lines. The agreement between the lattice and the continuum condensates at low magnetic fields again shows that the low- $\alpha$ part of the Hofstadter spectrum represents continuum physics. In fact, irrespective of how low the mass is, the initial segment of the lattice condensate is always smooth and follows the dependence dictated by the continuum Landau levels. The leading-order dependence of the condensate on the magnetic field (for non-vanishing masses) is quadratic in $B$. The corresponding coefficient - as demonstrated by explicit calculation in App. A - is proportional to the lowest-order coefficient $\beta_{1}=1 /\left(12 \pi^{2}\right)$ of the $\beta$-function of four-dimensional quantum electrodynamics (QED),

$$
\Delta \bar{\psi} \psi^{2 \mathrm{D}}=(q B)^{2} \cdot \beta_{1} \cdot \frac{4 \pi}{m^{3}}+\mathcal{O}\left(B^{4}\right)
$$

The sign of $\beta_{1}$ is fixed by the leading renormalization group behavior of the theory: the absence of asymptotic freedom in QED ensures that $\beta_{1}>0$. In turn, through Eq. (4.4) this results in a quadratic increase of the condensate with growing magnetic field. This observation leads to the following, three-fold correspondence:

wings of Hofstadter's butterfly at low magnetic fields

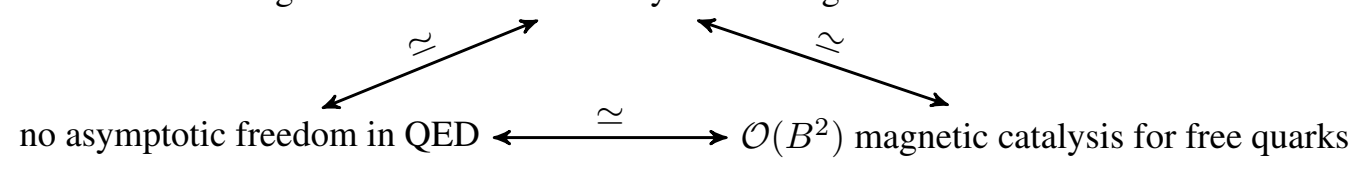

where 'magnetic catalysis' [10] refers to the enhancement of the condensate by $B$. I find this correspondence quite remarkable, as it connects three, seemingly unrelated phenomena: the spectrum in a solid state physics problem, a notion in perturbative quantum field theory and a characteristic about the breaking of chiral symmetry by the magnetic field.

Up to this point, the properties of the non-interacting Dirac operator were discussed. Let me now continue by making contact to the case of full QCD, considering the effect of gluonic interactions on the two-dimensional spectrum. In the presence of gluons, the $\mathrm{U}(1)$ links $u_{\mu}$ in the Dirac operator (3.9) are promoted to $\mathrm{SU}(3) \times \mathrm{U}(1)$ matrices $U_{\mu} \cdot u_{\mu}$. The interacting operator is diagonalized on an $x-y$ plane of a typical four-dimensional gluonic configuration. The resulting spectrum - together with the fermionic butterfly of the free case - is shown in Fig. 5. Apparently, QCD interactions tend to wash out the fractal structure and the Lan-

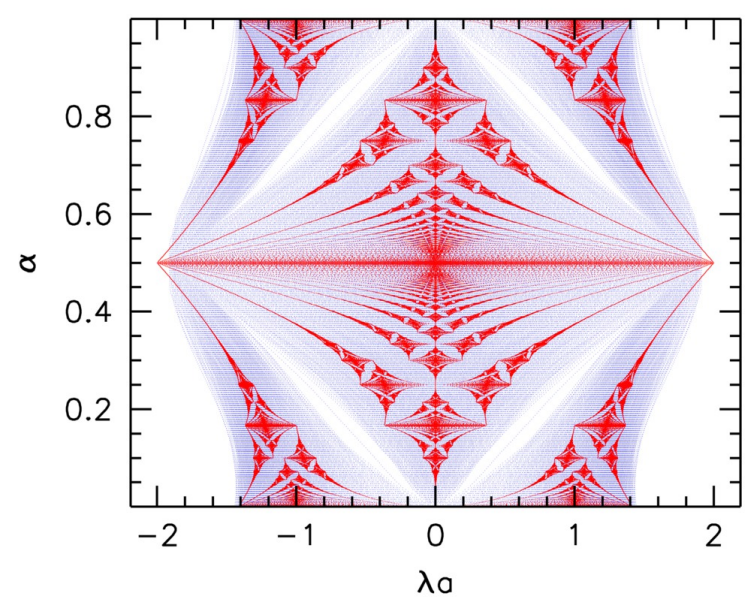

Figure 5: Fermionic butterfly in the free case (red) and with QCD interactions switched on (blue). 
dau levels composing the wings of the butterfly get smeared out. Nevertheless, the coarse structure of the spectrum - in particular the gap between the zeroth and first fermionic Landau levels - remains present in the interacting case as well. This qualitative similarity shows up in the dependence of the condensate on the magnetic field as well. Fig. 4 also includes $\Delta \bar{\psi} \psi^{2 \mathrm{D}}$ in the interacting case, featuring the quadratic increase of the condensate at low magnetic fields and the damped oscillations at larger values of $\alpha$.

As a side remark, I mention that the Fourier transform of the condensate with respect to $\alpha$ gives the so-called dual condensate, which can be used to calculate Wilson loops of fixed area [11], and thus makes contact to confinement in the interacting QCD case.

\section{Magnetic catalysis, inverse catalysis and the phase diagram}

Above I argued that the quadratic increase of the condensate of free quarks at low magnetic fields is related to the positivity of the QED $\beta$-function. (As the calculation in App. A shows, this holds both in two and in four dimensions.) The results for the condensate of interacting quarks (see Fig. 4) seem to indicate that the quadratic enhancement by $B$ is exhibited in the presence of gluons as well. Nevertheless, to determine the behavior of $\bar{\psi} \psi$ in full QCD, the complete QCD path integral has to be solved, for which large-scale numerical lattice simulations are necessary. Another approach that has often been followed is to simplify the problem by working with effective degrees of freedom or by approximating the theory by a model that e.g. captures the correct symmetries.

\subsection{Zero temperature}

The first results for the magnetic field-dependence of the quark condensate were obtained in such a model treatment, the Nambu-Jona-Lasinio (NJL) model $[12,10]$. In particular, it was found that the magnetic field always enhances the condensate, a mechanism that was dubbed magnetic catalysis [10]. This result was argued to be universal and model-independent. Indeed, $\bar{\psi} \psi$ was found to undergo magnetic catalysis in a host of different model and effective theory frameworks, see the recent review [13] and further references therein. The basic ingredient for magnetic catalysis was argued to lie in the dimensional reduction of the system for very strong magnetic fields [10]. The energy of the zeroth Landau level is $B$-independent (cf. Eq. (4.1)), whereas the next level lies much higher if $B$ is large, and its contribution to most physical observables is thus suppressed. In addition, the lowest Landau level effectively describes a one-dimensional system, since only the longitudinal momentum $p_{z}$ appears in it. This reduced dimensionality - together with the fact that the degeneracy of the levels is proportional to $B$ - leads to an increased phase space at low energies and thus supports the condensation of low eigenvalues. In turn, through the Banks-Casher relation [14], an increased density of the low eigenvalues translates to an enhancement of the quark condensate. Notice that this mechanism operates at high magnetic fields and is thus complementary to the arguments about the $\mathcal{O}\left(B^{2}\right)$ dependence of the condensate discussed in Eq. (4.5).

After the first model studies, numerical lattice simulations have also been performed to investigate the effect of magnetic fields on the condensate, see the review [17]. Magnetic catalysis was confirmed in the quenched theory [18], as well as in dynamical simulations with larger-thanphysical pion masses with three [19, 20] and with two colors [21]. In Refs. [22, 23] we simulated 

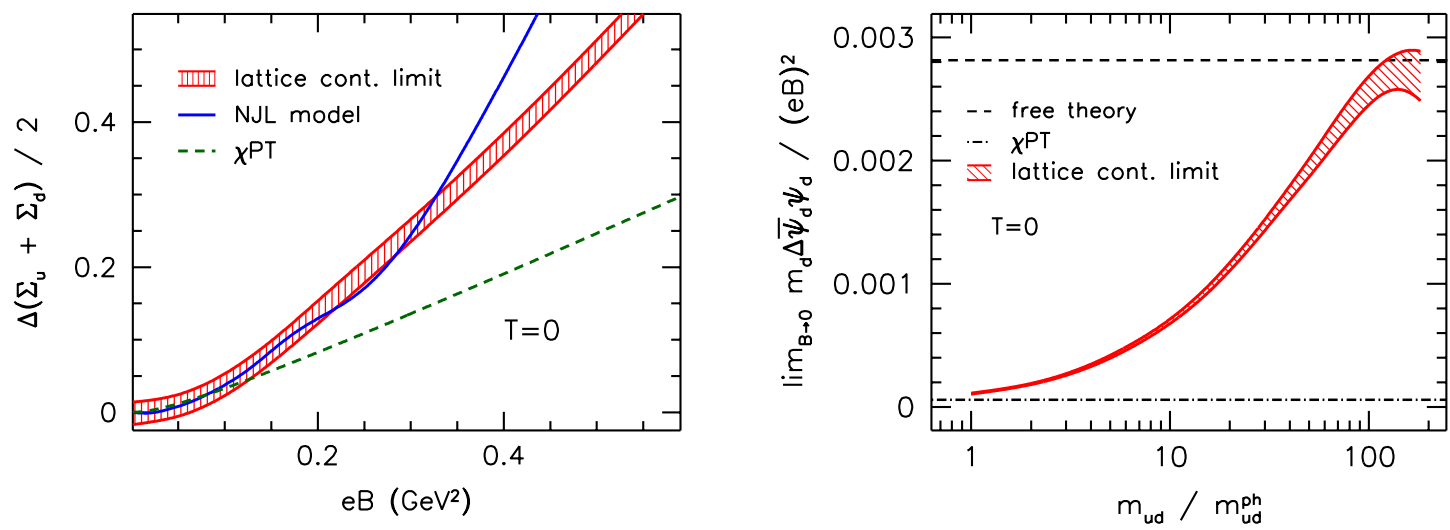

Figure 6: Left panel: the continuum limit of the lattice results for change of the condensate, compared to $\chi \mathrm{PT}$ [15] and to the NJL model prediction [16]. Right panel: the dependence of the coefficient of the quadratic contribution to the condensate, as a function of the quark mass. The continuum extrapolated lattice results interpolate between the limiting values, given by the free case $(m \rightarrow \infty)$ and by $\chi \mathrm{PT}(m \rightarrow 0)$.

full QCD with staggered quarks at physical masses and also performed the extrapolation of the results to the continuum limit. The average of the up and down quark condensates at $T=0$ is plotted in the left panel of Fig. 6 and also compared to chiral perturbation theory $(\chi \mathrm{PT})$ and to the NJL model, showing a qualitative - and, for low $B$, even quantitative - agreement. In summary, it is safe to say that at zero temperature all approaches agree and give a quark condensate that monotonously increases as $B$ grows.

To quantify the strength of magnetic catalysis, in Ref. [24] we also determined the coefficient of the quadratic enhancement of $\bar{\psi} \psi(B)$ for various values of the quark masses. On the one hand, for asymptotically high masses quarks and gluons decouple and the condensate can be calculated neglecting gluonic interactions (see App. A). On the other hand, if the mass approaches zero, $\chi \mathrm{PT}$ may be employed. Then, to leading order the condensate is calculated assuming free charged pions as effective degrees of freedom. The corresponding coefficient is again related to the $\beta$-function, but since pions are spinless, this time the scalar QED $\beta$-function coefficient $\beta_{1}^{\text {scalar }}=1 /\left(48 \pi^{2}\right)$ appears. In the two limits we get $[25,24]$

$$
\lim _{B \rightarrow 0} m \cdot \frac{\Delta \bar{\psi} \psi}{(e B)^{2}}=(q / e)^{2} \cdot \begin{cases}N_{c} \cdot \beta_{1}, & m \rightarrow \infty \\ \beta_{1}^{\text {scalar }} / 4, & m \rightarrow 0,\end{cases}
$$

where $N_{c}=3$ denotes the number of colors. As visible in the right panel of Fig. 6, the lattice results are completely consistent with this expectation and smoothly interpolate between the two extremes as the quark mass is varied.

\subsection{Nonzero temperature}

Most of the above mentioned models and effective theories predicted magnetic catalysis to be dominant for all temperatures $T$, both in the confined and in the deconfined phase of QCD. This uniform enhancement of the condensate also implied that the magnetic field shifts the restoration of chiral symmetry to higher temperatures, i.e. that $T_{c}(B)$ increases. Results obtained within various different frameworks seemed to point towards this conclusion, see the reviews [26]. In addition, lattice simulations with larger-than-physical quark masses also supported this picture [19, 21]. 
In contrast, in the large scale study in Refs. [22, 23] employing staggered quarks with physical masses and a continuum extrapolation, we found that magnetic catalysis gets weaker as the temperature increases, and in the transition region the condensate is even reduced by the magnetic field, see the left panel of Fig. 7. This behavior - that we dubbed inverse magnetic catalysis - also implied that chiral symmetry restoration occurs at lower temperatures as $B$ grows, i.e. that $T_{c}(B)$ decreases. The resulting phase diagram is plotted in the right panel of Fig. 7.
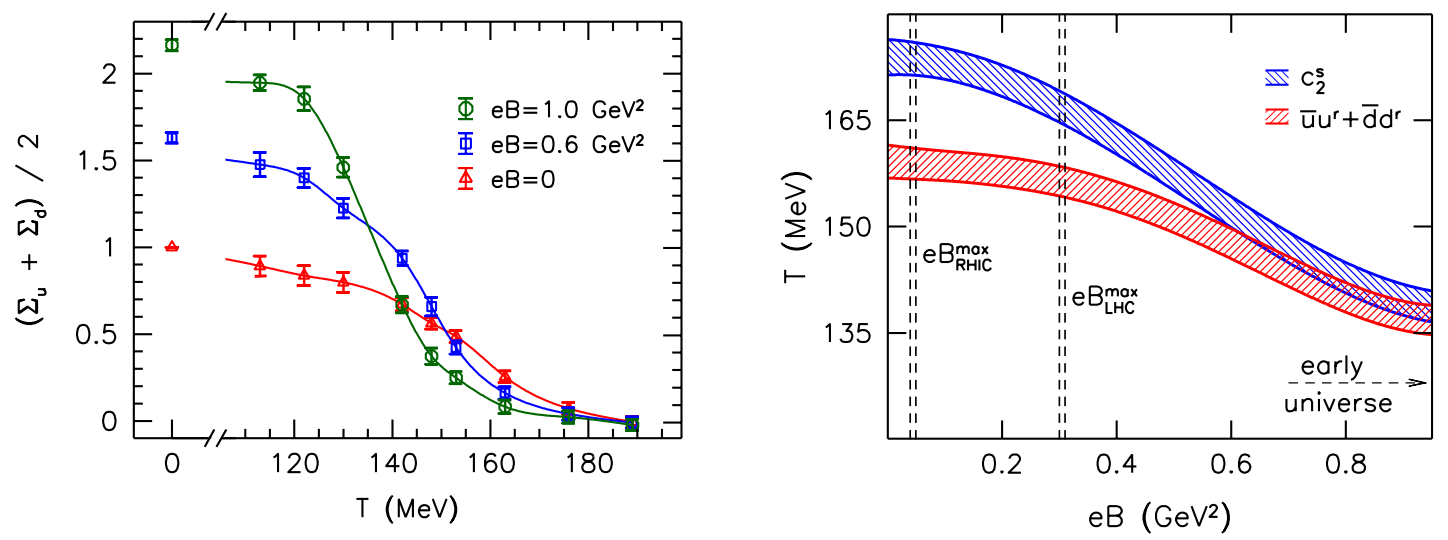

Figure 7: Left panel: magnetic catalysis at low temperatures and inverse magnetic catalysis in the transition region. As a result, the transition temperature - identified by the inflection point of the condensate - is decreased by $B$. Right panel: the QCD phase diagram in the $B-T$ plane, with $T_{c}$ defined as the inflection point of the quark condensate (red band) and as that of the strange quark number susceptibility (blue band).

In Ref. [22] we identified two reasons why inverse catalysis was not observed in earlier lattice simulations [19]: large cutoff effects and larger-than-physical quark masses. In fact, we explicitly demonstrated that inverse catalysis is only active for the light flavors and disappears for the heavier strange quark. In a follow-up paper [27] we discussed the possible mechanisms behind inverse catalysis. It turns out that the magnetic field induces two different contributions to the condensate: one that originates from the direct interaction between $B$ and valence quarks, and one that stems from the indirect coupling between $B$ and gluons [20]. In the path integral language, the former corresponds to the operator insertion, whereas the latter to the fermionic determinant. Separating these contributions revealed that the direct term always enhances the condensate, while the indirect contribution reduces it in the transition region. If the quark mass is small, the indirect effect turns out to dominate and induces inverse catalysis around $T_{c}$ [27]. From the perturbative point of view, the indirect effect may be described by the coupling of the background magnetic field (i.e. external photons) to virtual sea quark loops that interact with gluons. Due to the sea quark propagators, this diagram is proportional to $1 / \mathrm{m}^{2}$, revealing why it can only dominate for light quarks.

We then proceeded to identify the relevant gluonic degrees of freedom that this indirect effect couples to. The most important gauge degree of freedom around $T_{c}$ is the Polyakov loop, the pathordered parallel transport $P=\operatorname{Tr} \mathcal{P} \exp \left(\int_{0}^{1 / T} A_{4} d t\right)$ winding around the temporal direction of the lattice. Its dependence on the temperature and on the magnetic field is shown in Fig. 8. The results show that $P$ is drastically enhanced by the magnetic field in the transition region. This implies that the inflection point of $P$ is shifted to lower temperatures, revealing that around $T_{c}$ the magnetic 
field favors gauge configurations corresponding to the deconfined phase, i.e. with small condensate. This leads eventually to the inverse catalysis of $\bar{\psi} \psi$ in the transition region.

In addition, we have recently determined various thermodynamic observables related to the QCD equation of state [24] and found that these also support the reduction of $T_{c}$ by the magnetic field. Lattice results indicating this tendency have since been obtained using the overlap quark discretization in $N_{f}=2$ QCD [28] and in two-color QCD with four equally charged staggered quark flavors [29]. Besides the relevance of these findings for the physics of off-

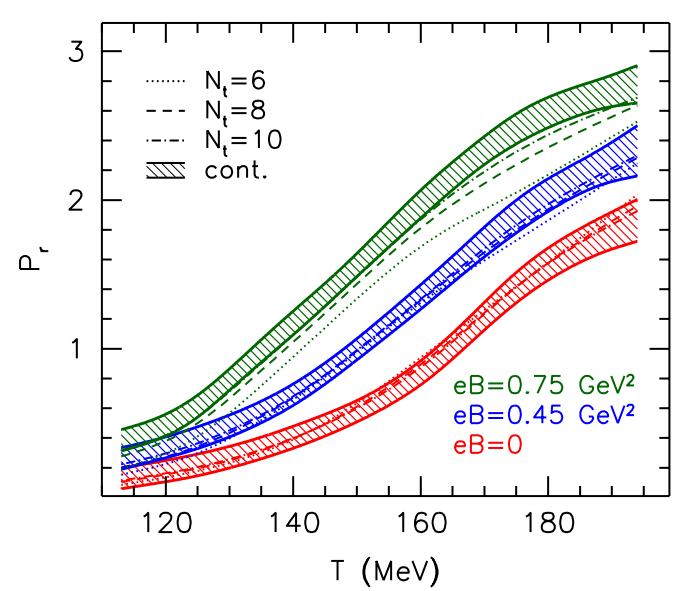

Figure 8: The Polyakov loop as a function of the temperature, for various values of $B$.

central heavy-ion collisions and early universe cosmology, the results have been highly useful for improving low-energy models of QCD. After the first attempts using simple models like the bag model [30] or large $N_{c}$ arguments [31], there is extensive ongoing work to implement inverse catalysis in different frameworks, e.g. by tuning the free model parameters to fit the lattice results or by looking for different mechanisms [32].

\section{Summary}

In the first part of the talk, I discussed the lattice Dirac eigenvalues of free quarks exposed to a background magnetic field in two dimensions. The spectrum - after a simple shift in the magnetic field - coincides with Hofstadter's butterfly. Although the butterfly is a mere lattice artefact and is eliminated in the continuum limit, the low- $B$ behavior of the eigenvalues does represent continuum physics. In particular, I derived the correspondence (4.5) between three, seemingly independent phenomena: a) the structure of the wings of Hofstadter's butterfly at small $B, b$ ) the absence of asymptotic freedom in QED and c) the leading-order magnetic catalysis of the quark condensate.

In the second part I turned to full QCD in four dimensions. Towards the chiral limit, chiral perturbation theory can be used to relate the $\mathcal{O}\left(B^{2}\right)$ magnetic catalysis to the positivity of the QED $\beta$-function (in this case the scalar QED $\beta$-function coefficient appears). Continuum extrapolated lattice results also confirm this scenario at zero temperature. For temperatures around $T_{c}$, however, gluonic interactions tend to reduce the condensate and induce inverse magnetic catalysis. This mechanism operates via the indirect interaction between the magnetic field and the gauge degrees of freedom (most importantly, the Polyakov loop) via charged sea quark loops. As a result, the transition temperature decreases as $B$ grows and the phase diagram looks as depicted on the right panel of Fig. 7. Progress has been made recently to implement such an indirect coupling in lowenergy models of QCD.

\section{A. Condensate in magnetic fields}

To reveal the role played by the QED $\beta$-function in the leading magnetic field-dependence of 
the condensate, it is instructive to first consider the free energy density $f$ of the system. To enable a direct comparison between the four- and the two-dimensional cases, the system dimension $2 \leq D \leq 4$ is not yet specified. At zero temperature, the logarithm of the partition function,

$$
\log \mathcal{Z}=\frac{1}{2} \oiint_{D} \log \lambda_{D}^{2}
$$

is written in terms of the $D$-dimensional energy eigenvalues and degeneracies

$$
\lambda_{D}^{2}=m^{2}+2 q B(n+1 / 2-s)+\sum_{i=3}^{D} p_{i}^{2}, \quad \oint_{D}=2 \cdot \frac{\Phi}{2 \pi} \sum_{n=0}^{\infty} \sum_{s= \pm 1 / 2} \prod_{i=3}^{D} L_{i} \int \frac{\mathrm{d} p_{i}}{2 \pi},
$$

where $\Phi=q B L_{x} L_{y}$ is the flux of the magnetic field. To regularize the sums and integrals the $\zeta$ function regularization (Mellin transform) is used ${ }^{3}$,

$$
\log \lambda_{D}^{2}=-\left.\frac{\partial}{\partial \alpha}\right|_{\alpha=0^{+}}\left(\lambda_{D}^{2}\right)^{-\alpha}, \quad\left(\lambda_{D}^{2}\right)^{-\alpha}=\frac{1}{\Gamma(\alpha)} \int_{0}^{\infty} \mathrm{d} z z^{\alpha-1} e^{-\lambda_{D}^{2} z}, \quad \operatorname{Re} \lambda_{D}^{2}>0 .
$$

Inserting this regularization, all sums and integrals can be performed. Differentiating with respect to $\alpha$,

$$
\left.\frac{\partial}{\partial \alpha}\right|_{\alpha=0^{+}} \frac{z^{\alpha}}{\Gamma(\alpha)}=1
$$

results in Schwinger's proper time representation [33] for the free energy density,

$$
f \equiv-\frac{1}{V_{D}} \log \mathcal{Z}=\frac{2 q B}{(4 \pi)^{D / 2}} \int_{0}^{\infty} \frac{\mathrm{d} z}{z^{D / 2}} e^{-m^{2} z} \operatorname{coth}(q B z),
$$

where $V_{D}=\prod_{i} L_{i}$ is the $D$-dimensional volume.

To obtain the leading dependence on the magnetic field, $\Delta f \equiv f(B)-f(B=0)$ is expanded in $q B$,

$$
\Delta f=\frac{(q B)^{2}}{m^{4-D}} \frac{2}{3(4 \pi)^{D / 2}} \Gamma(2-D / 2)+\mathcal{O}\left(B^{4}\right) .
$$

The $\mathcal{O}\left(B^{2}\right)$ term in the free energy density corresponds to the coupling of a fermion loop to two external photon legs (the external photons represent the background magnetic field), i.e. the photon vacuum polarization diagram $[33,34,35]$. In $D=4$ dimensions, this diagram is logarithmically divergent and its coefficient equals the lowest-order QED $\beta$-function coefficient $\beta_{1}=1 /\left(12 \pi^{2}\right)$. In $D=2$ dimensions, the vacuum polarization is ultraviolet finite, but the coefficient is still proportional to $\beta_{1}$,

$$
\Delta f=\frac{(q B)^{2}}{2} \cdot \beta_{1} \cdot\left\{\begin{array}{ll}
\frac{1}{\varepsilon}-\gamma-\log m^{2}+\mathcal{O}(\varepsilon), & D=4-2 \varepsilon \\
4 \pi / m^{2}, & D=2
\end{array}+\mathcal{O}\left(B^{4}\right)\right.
$$

where $\gamma$ is Euler's constant.

Differentiating Eq. (A.5) with respect to the mass gives the condensate,

$$
\bar{\psi} \psi \equiv-\frac{\partial f}{\partial m}=\frac{4 m q B}{(4 \pi)^{D / 2}} \int_{0}^{\infty} \frac{\mathrm{d} z}{z^{D / 2-1}} e^{-m^{2} z} \operatorname{coth}(q B z),
$$

\footnotetext{
${ }^{3}$ I would like to thank Falk Bruckmann for the countless discussions that we had about this and similar calculations.
} 
which, after a similar expansion in the magnetic field as above, gives

$$
\Delta \bar{\psi} \psi=-\frac{\partial \Delta f}{\partial m}=(q B)^{2} \cdot \beta_{1} \cdot\left\{\begin{array}{ll}
1 / m, & D=4 \\
4 \pi / m^{3}, & D=2
\end{array}+\mathcal{O}\left(B^{4}\right) .\right.
$$

Notice that the difference between $D=4$ and $D=2$ is the different power of the mass to account for the dimensionality, and the factor $4 \pi$ that stems from the additional phase space in the third and fourth dimensions. Altogether, Eq. (A.9) shows that the positivity of $\beta_{1}-$ i.e., the absence of asymptotic freedom in QED - results in an $\mathcal{O}\left(B^{2}\right)$ increase of the condensate of free quarks, both in two and in four dimensions. This argument has been used in Refs. [25, 36, 24] to relate magnetic catalysis to the positivity of the QED $\beta$-function for $D=4$. Note that for quarks the number $N_{c}$ of colors also appears in Eq. (A.9) as a multiplicative factor.

\section{References}

[1] A. S. Kronfeld PoS LATTICE2013 (2013) 504, [arXiv:1312 . 6861]; P. Ginsparg arXiv:1407.1855.

[2] D. Kharzeev, K. Landsteiner, A. Schmitt, and H.-U. Yee Lect.Notes Phys. 871 (2013) 1-624.

[3] D. R. Hofstadter Phys.Rev. B14 (1976) 2239-2249.

[4] Y. Last Communications in Mathematical Physics 164 (1994), no. 2 421-432.

[5] G. Martinelli, G. Parisi, R. Petronzio, and F. Rapuano Phys. Lett. B116 (1982) 434.

[6] G. 't Hooft Nucl. Phys. B153 (1979) 141.

[7] T. Kimura arXiv:1210.6355.

[8] C. R. Dean et al. Nature 497 (May, 2013) 598-602; L. A. Ponomarenko et al. Nature 497 (May, 2013) 594-597, [arXiv:1212.5012].

[9] The eigenvalues and the program to illustrate the mass-dependence of the condensate can be found at homepages.uni-regensburg.de/ eng14891/finiteTQFT/hofstadter.tar.gz.

[10] V. P. Gusynin, V. A. Miransky, and I. A. Shovkovy Nucl. Phys. B 462 (1996) 249, [hep-ph/9509320].

[11] F. Bruckmann and G. Endrődi Phys.Rev. D84 (2011) 074506, [arXiv:1104. 5664].

[12] S. Schramm, B. Müller, and A. J. Schramm Mod.Phys.Lett. A7 (1992) 973-982.

[13] I. A. Shovkovy Lect.Notes Phys. 871 (2013) 13-49, [arXiv:1207. 5081].

[14] T. Banks and A. Casher Nucl.Phys. B169 (1980) 103.

[15] T. D. Cohen, D. A. McGady, and E. S. Werbos Phys. Rev. C 76 (2007) 055201, [arXiv:0706.3208].

[16] R. Gatto and M. Ruggieri Phys. Rev. D 83 (2011) 034016, [arXiv:1012.1291].

[17] M. D'Elia Lect.Notes Phys. 871 (2013) 181-208, [arXiv: 1209 . 0374].

[18] P. Buividovich, M. Chernodub, E. Luschevskaya, and M. Polikarpov Phys. Lett. B 682 (2010) 484, [arXiv:0812.1740]. 
[19] M. D’Elia, S. Mukherjee, and F. Sanfilippo Phys. Rev. D 82 (2010) 051501, [a rXiv: 1005.5365 ].

[20] M. D’Elia and F. Negro Phys. Rev. D 83 (2011) 114028, [arXiv:1103.2080].

[21] E.-M. Ilgenfritz, M. Kalinowski, M. Müller-Preussker, B. Petersson, and A. Schreiber Phys.Rev. D85 (2012) 114504, [arXiv:1203.3360].

[22] G. Bali, F. Bruckmann, G. Endrődi, Z. Fodor, S. Katz, S. Krieg, A. Schäfer, and K. Szabó JHEP 2012 (2012) 1, [arXiv:1111.4956].

[23] G. Bali, F. Bruckmann, G. Endrődi, Z. Fodor, S. Katz, et al. Phys.Rev. D86 (2012) 071502 , [arXiv:1206.4205].

[24] G. Bali, F. Bruckmann, G. Endrődi, S. Katz, and A. Schäfer JHEP 1408 (2014) 177, [arXiv:1406.0269].

[25] G. Endrődi JHEP 1304 (2013) 023, [arXiv: 1301.1307$].$

[26] E. S. Fraga Lect.Notes Phys. 871 (2013) 121-141, [arXiv:1208.0917]; R. Gatto and M. Ruggieri Lect.Notes Phys. 871 (2013) 87-119, [arXiv:1207.3190].

[27] F. Bruckmann, G. Endrődi, and T. G. Kovács JHEP 1304 (2013) 112, [arXiv: 1303.3972 ].

[28] V. Bornyakov, P. Buividovich, N. Cundy, O. Kochetkov, and A. Schäfer Phys.Rev. D90 (2014) 034501, [arXiv:1312.5628].

[29] E. M. Ilgenfritz, M. Müller-Preussker, B. Petersson, and A. Schreiber Phys.Rev. D89 (2014) 054512, [arXiv:1310.7876].

[30] E. S. Fraga and L. F. Palhares Phys.Rev. D86 (2012) 016008, [arXiv:1201.5881].

[31] E. S. Fraga, J. Noronha, and L. F. Palhares Phys.Rev. D87 (2013), no. 11114014 , [arXiv:1207.7094].

[32] K. Fukushima and Y. Hidaka Phys.Rev.Lett. 110 (2013) 031601, [arXiv:1209.1319]; J. Chao, P. Chu, and M. Huang Phys.Rev. D88 (2013) 054009, [arXiv:1305.110 0]; E. Fraga, B. Mintz, and J. Schaffner-Bielich Phys.Lett. B731 (2014) 154-158, [arXiv:1311.3964]; M. Ferreira, P. Costa, D. P. Menezes, C. Providência, and N. Scoccola Phys.Rev. D89 (2014) 016002, [arXiv: 1305. 4751]; K. Kamikado and T. Kanazawa JHEP 1403 (2014) 009, [arXiv:1312.3124]; E. Ferrer, V. de la Incera, and X. Wen arXiv:1407.3503; S. Fayazbakhsh and N. Sadooghi arXiv: 1408.5457 ; R. Farias, K. Gomes, G. Krein, and M. Pinto arXiv: 1404 .3931; M. Ferreira, P. Costa, O. Lourenço, T. Frederico, and C. Providência Phys.Rev. D89 (2014) 116011, [arXiv:1404.5577]; A. Ayala, M. Loewe, A. J. Mizher, and R. Zamora Phys.Rev. D90 (2014) 036001, [arXiv:1406.3885]; A. Ayala, M. Loewe, and R. Zamora arXiv:1406.7408; J. O. Andersen, W. R. Naylor, and A. Tranberg arXiv:1410.5247.

[33] J. S. Schwinger Phys.Rev. 82 (1951) 664-679.

[34] L. Abbott Acta Phys.Polon. B13 (1982) 33.

[35] G. V. Dunne hep-th/0406216.

[36] G. Bali, F. Bruckmann, G. Endrődi, and A. Schäfer PoS LATTICE2013 (2013) 182, [arXiv:1310.8145]. 